\title{
ON A GENERALIZATION OF THE NOTION OF CENTRALIZING MAPPINGS
}

\author{
MATEJ BRESAR
}

(Communicated by Maurice Auslander)

\begin{abstract}
Let $R$ be a ring with center $Z$. A mapping $f: R \rightarrow R$ is called centralizing (resp. commuting) if $[f(x), x] \in Z$ (resp. $[f(x), x]=0)$ for all $x \in R$. In this paper we consider a more general case where a mapping $f: R \rightarrow R$ satisfies $[[f(x), x], x]=0$ for all $x \in R$; it is shown that if $R$ is a prime ring of characteristic not 2 , then every additive mapping with this property is commuting.
\end{abstract}

Let $R$ be a ring with center $Z$. A mapping $f$ of $R$ into itself is called centralizing if $[f(x), x] \in Z$ for all $x \in R$, where $[u, v]$ denotes the commutator $u v-v u$. In the special case where $[f(x), x]=0$ for all $x \in R$, the mapping $f$ is said to be commuting. In [10] Posner initiated the study of centralizing mappings. He showed that if $d$ is a centralizing derivation of a prime ring $R$, then either $d=0$ or $R$ is commutative. Over the last twenty years a lot of work has been done on centralizing and commuting mappings. We refer the reader to some recent papers $[1,2,3,4,5,7,9,11]$ where further references can be found.

Recently Vukman [11] extended Posner's theorem by showing that if $d$ is a derivation of a prime ring $R$ of characteristic not 2 , such that $[[d(x), x], x]=$ 0 for all $x \in R$, then $d=0$ or $R$ is commutative. In fact, in view of Posner's theorem he merely showed that $d$ is commuting. It is our aim in this paper to prove that this conclusion holds for any additive mapping. More precisely, we prove the following result.

Theorem 1. Let $R$ be a prime ring of characteristic not 2 . If an additive mapping $f: R \rightarrow R$ satisfies $[[f(x), x], x]=0$ for all $x \in R$, then $[f(x), x]=0$ for all $x \in R$ (i.e., $f$ is commuting).

In particular, this theorem implies that every additive centralizing mapping of a prime ring of characteristic not 2 is actually commuting. However, in our forthcoming paper [4] it is shown that this result is true under some weaker hypothesis. In [4] we also proved the following result: If $f$ is an additive commuting mapping of a prime ring $R$, then there exist an element $\lambda$ in $C$, the

Received by the editors September 27, 1990.

1980 Mathematics Subject Classification (1985 Revision). Primary 16A12, 16A70, 16A72. troid.

Key words and phrases. Centralizing mapping, commuting mapping, prime ring, extended cen- 
extended centroid of $R$, and an additive mapping $\zeta: R \rightarrow C$, such that $f(x)=$ $\lambda x+\zeta(x)$ for all $x \in R$. Combining this result with Theorem 1, one obtains a characterization of additive mappings $f: R \rightarrow R$ satisfying $[[f(x), x], x]=0$ for all $x \in R$, where $R$ is a prime ring of characteristic not 2 .

We also remark that in view of Theorem 1, several results in papers quoted above can now be stated in a more general form.

The statement of Theorem 1 can be expressed as follows: If $R$ is a prime ring of characteristic not 2 and $f: R \rightarrow R$ is an additive mapping such that the mapping $x \rightarrow[f(x), x]$ is commuting, then $f$ is commuting. The question arises whether the same conclusion holds if we assume that the mapping $x \rightarrow[f(x), x]$ is centralizing. Neglecting the fact that we have the additional assumption that $R$ is 3-torsionfree, the following result gives much more than the affirmative answer to this question.

Theorem 2. Let $R$ be a ring, and let $B: R \times R \rightarrow R$ be a biadditive mapping. Suppose that the mapping $x \rightarrow B(x, x)$ is centralizing. If $R$ is 2-torsionfree and 3-torsionfree, and if the center $Z$ of $R$ does not contain any nonzero nilpotents (in particular, if $R$ is semiprime), then the mapping $x \rightarrow B(x, x)$ is commuting.

Of course, Theorem 2 can be applied to the case where $f: R \rightarrow R$ is an additive mapping with the property that the mapping $x \rightarrow[f(x), x]$ is centralizing. Combining this fact with Theorem 1 we get

Corollary 1. Let $R$ be a prime ring of characteristic different from 2 and 3. If $f: R \rightarrow R$ is an additive mapping, such that the mapping $x \rightarrow[f(x), x]$ is centralizing, then $f$ is commuting.

As an immediate consequence of Corollary 1 and Posner's theorem, we obtain the main result in Vukman's paper [11], which states that if $R$ is a noncommutative prime ring of characteristic different from 2 and 3 and $d$ is a derivation of $R$ such that the mapping $x \rightarrow[d(x), x]$ is centralizing, then $d=0$.

The proof of Theorem 2 is entirely elementary, while our method in proving Theorem 1 requires the use of some results on Martindale's extended centroid. Therefore we first recall a few facts concerning extended centroid and central closure of a prime ring (see [6] or [8] for details). Let $R$ be a prime ring, and let $M$ be the set of all pairs $(U, f)$, where $U$ is a nonzero ideal of $R$ and $f: U \rightarrow R$ is a right $R$-module map of $U$ into $R$. We define an equivalence relation on $M$ by $(U, f) \sim(V, g)$ if $f=g$ on some nonzero ideal $W$ contained in $U \cap V$. The set $Q$ of all equivalence classes forms a ring under the operations induced by addition and composition of representatives of the equivalence classes. We embed $R$ in $Q$ via the left multiplications on $R$ by the elements in $R$. The center $C$ of $Q$ is a field containing the centroid of $R$ and is called the extended centroid of $R$. The $C$-algebra $S=R C+C$ is again a prime ring and is called the central closure of $R$. The extended centroid of $S$ is $C$. Thus the center of $S$ coincides with the extended centroid of $S$.

We make crucial use of the following result.

Lemma 1 [6, Lemma 1.3.2]. Suppose that elements $a_{i}, b_{i}$ in $R$ satisfy $\sum a_{i} x b_{i}=$ 0 for all $x \in R$. If the $b_{i}$ 's are nonzero then the $a_{i}$ 's are linearly independent over $C$.

We also need 
Lemma 2. If $s \in S$ satisfies $[[s, x], x]=0$ for all $x \in R$, then $s \in C$.

If $s \in R$, then Lemma 2 is merely a special case of Posner's theorem. Fortunately, even if $s \notin R$ the same proof works, but we include it for the sake of completeness.

Proof of Lemma 2. A linearization of $[[s, x], x]=0$ gives

$$
[[s, x], y]+[[s, y], x]=0 \text { for all } x, y \in R \text {. }
$$

Replacing $y$ by $y x$ in (1) and using $[[s, x], x]=0$, we obtain

$$
\begin{aligned}
0 & =[[s, x], y x]+[[s, y x], x]=[[s, x], y] x+[[s, y] x+y[s, x], x] \\
& =[[s, x], y] x+[[s, y], x] x+[y, x][s, x] .
\end{aligned}
$$

Applying (1), we then get $[y, x][s, x]=0$ for all $x, y \in R$. Taking $y z$ for $y$ in this relation and using $[y z, x]=[y, x] z+y[z, x]$, we see that $[y, x] R[s, x]=0$ for all $x, y \in R$. But then also $[s, x] S[s, x]=0$ for all $x \in R$. Since $S$ is prime, it follows that $s$ commutes with all elements in $R$; hence $s \in C$.

We now have enough information to prove Theorem 1.

Proof of Theorem 1. For the proof we need several steps. In the first lemma, we extend the mapping $(x, y) \rightarrow[f(x), y]$, where $x, y \in R$, to a biadditive mapping of $S \times S$ to $S$.

Lemma A. There exists a biadditive mapping $B: S \times S \rightarrow S$ such that $B(x, y)=$ $[f(x), y]$ for $x, y \in R$. Moreover, $B$ has the following properties:

(i) $B(s, \lambda)=B(\lambda, s)=0$ for all $\lambda \in C, s \in S$;

(ii) for $t \in S$, the mapping $s \rightarrow B(t, s)$ is an inner derivation;

(iii) $[B(s, s), s]=0$ for all $s \in S$.

Proof. Taking $x+y$ instead of $x$ in $[[f(x), x], x]=0$, we obtain

(2) $\quad[[f(x), x], y]+[[f(x), y], x]+[[f(x), y], y]$

$$
+[[f(y), x], x]+[[f(y), x], y]+[[f(y), y], x]=0
$$

for all $x, y \in R$. Replacing $y$ by $-y$ in (2), and comparing the relation so obtained with (2), we obtain, since $R$ has a characteristic different from 2 , that

$$
[[f(x), x], y]+[[f(x), y], x]+[[f(y), x], x]=0 \text { for all } x, y \in R
$$

This relation is needed in the sequel. Now define $B: S \times S \rightarrow S$ by

$$
B\left(\sum_{i=1}^{n} \lambda_{i} x_{i}+\mu, s\right)=\left[\sum_{i=1}^{n} \lambda_{i} f\left(x_{i}\right), s\right] .
$$

In order to show that $B$ is well defined, suppose $\sum_{i=1}^{n} \lambda_{i} x_{i}+\mu=0$. We must verify that in this case, the element $\sum_{i=1}^{n} \lambda_{i} f\left(x_{i}\right)$ commutes with all elements in $S$. We may assume that $\lambda_{1} \neq 0$. Hence $x_{1}=\sum_{i=2}^{n} \mu_{i} x_{i}+\nu$ where $\mu_{i}=-\lambda_{1}^{-1} \lambda_{i}$ 
and $\nu=-\lambda_{1}^{-1} \mu$. By (3) it follows that for any $x \in R$, we have

$$
\begin{aligned}
{\left[x,\left[f\left(x_{1}\right), x\right]\right] } & =\left[[f(x), x], x_{1}\right]+\left[\left[f(x), x_{1}\right], x\right] \\
& =\left[[f(x), x], \sum_{i=2}^{n} \mu_{i} x_{i}+\nu\right]+\left[\left[f(x), \sum_{i=2}^{n} \mu_{i} x_{i}+\nu\right], x\right] \\
& =\sum_{i=2}^{n} \mu_{i}\left\{\left[[f(x), x], x_{i}\right]+\left[\left[f(x), x_{i}\right], x\right]\right\} \\
& =\sum_{i=2}^{n} \mu_{i}\left[x,\left[f\left(x_{i}\right), x\right]\right]=\left[x,\left[\sum_{i=2}^{n} \mu_{i} f\left(x_{i}\right), x\right]\right] .
\end{aligned}
$$

Thus

$$
\left[x,\left[f\left(x_{1}\right)-\sum_{i=2}^{n} \mu_{i} f\left(x_{i}\right), x\right]\right]=0 \text { for all } x \in R .
$$

By Lemma 2 it follows that the element $f\left(x_{1}\right)-\sum_{i=2}^{n} \mu_{i} f\left(x_{i}\right)$ is contained in $C$. But then also $\sum_{i=1}^{n} \lambda_{i} f\left(x_{i}\right) \in C$. This means that $B$ is well defined.

It is obvious that $B$ is a biadditive mapping satisfying (i) and (ii). Using (3) and its linearized form, one shows by a direct computation that $B$ also satisfies (iii). Lemma $\mathrm{A}$ is thereby proved.

We assume henceforth that $S$ is any prime ring of characteristic not 2, such that its center $C$ coincides with its extended centroid, and that $B: S \times S \rightarrow S$ is an additive mapping satisfying (i), (ii), and (iii). Our intention is to show that $B(s, s)=0$ for every $s$ in $S$. According to Lemma A, with this the theorem is proved.

First note that the same approach as in the proof of (3) also gives the following:

$$
[B(s, s), t]+[B(s, t), s]+[B(t, s), s]=0 \text { for all } s, t \in S .
$$

We continue with a technical lemma.

Lemma B. For all $u, s \in S$,

$$
u^{2} s B(u, u)=u s B\left(u^{2}, u\right)+s\left(u^{2} B(u, u)-u B\left(u^{2}, u\right)\right) .
$$

Proof. Define a mapping $D: S \times S \times S \rightarrow S$ by

$$
D(s, t, u)=[B(s, t), u]+[B(s, u)+B(u, s), t] .
$$

Linearizing (4) we see that on the other hand

$$
D(s, t, u)=[u, B(t, s)]+[s, B(t, u)+B(u, t)] .
$$

Given $u, s \in S$ we compute $D\left(s u, u^{2}, u\right)$ in two ways. The idea behind these computations is the observation that, in view of (5) and (ii), the mapping $t \rightarrow B(s, t, u)$ is equal to the sum of the composition of two derivations and the derivation; similarly, by (6) and (ii), the mapping $s \rightarrow B(s, t, u)$ has the same property.

According to (5) we have

$$
D\left(s u, u^{2}, u\right)=\left[B\left(s u, u^{2}\right), u\right]+\left[B(s u, u)+B(u, s u), u^{2}\right]
$$


By assumption, the mapping $t \rightarrow B(s u, t)$ is a derivation, so it follows that

$$
\begin{aligned}
D\left(s u, u^{2}, u\right)= & {[B(s u, u) u+u B(s u, u), u]+\left[B(s u, u)+B(u, s u), u^{2}\right] } \\
= & {[B(s u, u), u] u+u[B(s u, u), u]+[B(s u, u)+B(u, s u), u] u } \\
& +u[B(s u, u)+B(u, s u), u]
\end{aligned}
$$

by $(5)$ this relation can be written in the form

$$
D\left(s u, u^{2}, u\right)=D(s u, u, u) u+u D(s u, u, u) .
$$

We now look at the expression $D(s u, u, u)$. By (6) we have

$$
D(s u, u, u)=[u, B(u, s u)]+[s u, 2 B(u, u)] .
$$

Since the mapping $t \rightarrow B(u, t)$ is a derivation, it follows that

$$
D(s u, u, u)=[u, B(u, s) u+s B(u, u)]+[s u, 2 B(u, u)] .
$$

According to $[B(u, u), u]=0$, we then get

$$
\begin{aligned}
D(s u, u, u) & =[u, B(u, s)] u+[u, s] B(u, u)+[s, 2 B(u, u)] u \\
& =D(s, u, u) u+[u, s] B(u, u) .
\end{aligned}
$$

Using this relatin in (7), we obtain

$$
\begin{aligned}
D\left(s u, u^{2}, u\right)= & D(s, u, u) u^{2}+[u, s] B(u, u) u \\
& +u D(s, u, u) u+u[u, s] B(u, u) .
\end{aligned}
$$

The computation on the first way is thereby finished. We begin the computation on the second way by applying (6):

$$
D\left(s u, u^{2}, u\right)=\left[u, B\left(u^{2}, s u\right)\right]+\left[s u, B\left(u^{2}, u\right)+B\left(u, u^{2}\right)\right] .
$$

The mapping $t \rightarrow B\left(u^{2}, t\right)$ is a derivation, hence

(9) $D\left(s u, u^{2}, u\right)=\left[u, B\left(u^{2}, s\right) u+s B\left(u^{2}, u\right)\right]+\left[s u, B\left(u^{2}, u\right)+B\left(u, u^{2}\right)\right]$

Since $B\left(u, u^{2}\right)=B(u, u) u+u B(u, u)$ and since $[B(u, u), u]=0$, it follows that $\left[B\left(u, u^{2}\right), u\right]=0$. By $(4)$ we then also have $\left[B\left(u^{2}, u\right), u\right]=0$. Therefore (9) can be written in the form

$$
D\left(s u, u^{2}, u\right)=\left[u, B\left(u^{2}, s\right)\right] u+[u, s] B\left(u^{2}, u\right)+\left[s, B\left(u^{2}, u\right)+B\left(u, u^{2}\right)\right] u .
$$

Thus

$$
D\left(s u, u^{2}, u\right)=D\left(s, u^{2}, u\right) u+[u, s] B\left(u^{2}, u\right) .
$$

Using (5) we have

$$
\begin{aligned}
D\left(s, u^{2}, u\right)= & {\left[B\left(s, u^{2}\right), u\right]+\left[B(s, u)+B(u, s), u^{2}\right] } \\
= & {[B(s, u) u+u B(s, u), u]+\left[B(s, u)+B(u, s), u^{2}\right] } \\
= & {[B(s, u), u] u+u[B(s, u), u]+[B(s, u)+B(u, s), u] u } \\
& +u[B(s, u)+B(u, s), u] \\
= & D(s, u, u) u+u D(s, u, u)
\end{aligned}
$$

hence (10) yields

$$
D\left(s u, u^{2}, u\right)=D(s, u, u) u^{2}+u D(s, u, u) u+[u, s] B\left(u^{2}, u\right) .
$$


Comparing (8) and (11), we get

$$
[u, s] B(u, u) u+u[u, s] B(u, u)=[u, s] B\left(u^{2}, u\right) .
$$

Since $B(u, u) u=u B(u, u)$, this relation can be written in the form $u^{2} s B(u, u)$ $=u s B\left(u^{2}, u\right)+s\left(u^{2} B(u, u)-u B\left(u^{2}, u\right)\right)$, which proves the lemma.

We show that if $B(u, u) \neq 0$ for some $u \in S$, then $u$ is rather special.

Lemma C. If $u \in S$ and $B(u, u) \neq 0$, then there exists $\lambda \in C$ such that $(2 u-\lambda)^{2}=0$.

Proof. Since $B(u, u) \neq 0$, it follows from Lemmas B and 1 that there exist $\lambda_{1}, \lambda_{2}, \lambda_{3}$ not all zero in $C$ such that $\lambda_{1} u^{2}+\lambda_{2} u+\lambda_{3}=0$. Suppose that $\lambda_{1}=0$. Obviously in this case, $\lambda_{2}$ and $\lambda_{3}$ are different from zero, and so $\lambda_{2} u+\lambda_{3}=0$ implies that $u \in C$. But then $B(u, u)=0$ by (i). Thus we may assume that $\lambda_{1} \neq 0$. Hence $u^{2}=\lambda u+\mu$ where $\lambda=-\lambda_{1}^{-1} \lambda_{2} \in C$ and $\mu=-\lambda_{1}^{-1} \lambda_{3} \in C$.

By assumption, there exists $a \in S$ such that $B(u, t)=[a, t]$ for all $t \in S$. Thus the relation $[B(u, u), u]=0$ can be written in the form $a u^{2}+u^{2} a=2 u a u$. Since $u^{2}=\lambda u+\mu$ it follows that

$$
\lambda a u+2 \mu a+\lambda u a=2 u a u .
$$

Multiply (12) from the right by $u$, we then get

$$
\lambda a(\lambda u+\mu)+2 \mu a u+\lambda u a u=2 u a(\lambda u+\mu) .
$$

That is,

$$
\left(\lambda^{2}+2 \mu\right) a u+\lambda \mu a-2 \mu u a=\lambda u a u .
$$

Multiply the relation (10) by $\lambda$, the relation (11) by 2 , and compare the relations so obtained. Then we arrive at $\left(\lambda^{2}+4 \mu\right) a u=\left(\lambda^{2}+4 \mu\right) u a$, i.e., $\left(\lambda^{2}+\right.$ $4 \mu) B(u, u)=0$. Since we have assumed that $B(u, u) \neq 0$, it follows that $\lambda^{2}+4 \mu=0$. Hence the relation $u^{2}=\lambda u+\mu$ yields $(2 u-\lambda)^{2}=0$.

Lemma D. If $v \in S$ and $v^{2}=0$, then $B(v, v)=0$.

Proof. By assumption, for every $t \in S$ there exists $b(t) \in S$ such that $B(t, s)=$ $[b(t), s]$ for all $s \in S$. We denote the element $b(v)$ by $b$. Taking $v$ for $s$ in (4) we obtain

$$
[[b, v], t]+[[b, t], v]+[[b(t), v], v]=0 \text { for all } t \in S .
$$

Since $B(v, v)=[b, v]$ commutes with $v$ and since $v^{2}=0$, it follows that $2 v b v=0$; thus $v b v=0$ and therefore, also $v[b, v]=0$. Now multiply (14) from the left by $v$. Using $v^{2}=0$ and the last statement, we obtain

$$
-v t[b, v]+v[b, t] v=0 \text { for all } t \in S \text {. }
$$

This relation can be written in the form

$$
v b t v=v t(2 b v-v b) \quad \text { for all } t \in S .
$$

Replacing $t$ by $t v r$ in (15), we obtain $v b t v r v=v t v r(2 b v-v b)$. But on the other hand, again according to (15), we have $v b t v r v=v t(2 b v-v b) r v$ and $v t v r(2 b v-v b)=v t v b r v$. Comparing these relations, we get $v t(2 b v-2 v b) r v=$ 
0 where $t$ and $r$ are arbitrary elements in $S$. Therefore $2[b, v]=2 B(v, v)=$ 0 by the primeness of $S$. But then $B(v, v)=0$.

Now, suppose that $B(u, u) \neq 0$ for some $u \in S$. By Lemma $C$ there exists $\lambda \in C$ such that $(2 u-\lambda)^{2}=0$. Thus $B(2 u-\lambda, 2 u-\lambda)=0$ by Lemma D. But on the other hand, since $B$ is biadditive and since (i) holds, $B(2 u-\lambda, 2 u-\lambda)=4 B(u, u)$. Hence $B(u, u)=0$. With this contradiction, Theorem 1 is proved.

Proof of Theorem 2. Since $R$ is 2-torsionfree, we may assume that $B$ is symmetric (i.e., $B(x, y)=B(y, x)$ for $x, y \in R)$; otherwise replace $B$ by the mapping $(x, y) \rightarrow B(x, y)+B(y, x)$.

By assumption, for every $x \in R$ we have $[B(x, x), x] \in Z$. A linearization yields

$$
[B(x, x), y]+2[B(x, y), x]+2[B(x, y), y]+[B(y, y), x] \in Z .
$$

Replace $x$ by $-x$ in (16); comparing the relation so obtained with (16), we then get $2([B(x, x), y]+2[B(x, y), x]) \in Z$. Since $R$ is 2-torsionfree, it follows that

$$
[B(x, x), y]+2[B(x, y), x] \in Z \text { for all } x, y \in R .
$$

Now fix $x \in R$ and let us show that the element $c=[B(x, x), x] \in Z$ is equal to 0 . Take $x^{2}$ for $y$ in (17); since $\left[B(x, x), x^{2}\right]=[B(x, x), x] x+$ $x[B(x, x), x]=c x+x c=2 c x$, we then get $2 c x+2\left[B\left(x, x^{2}\right), x\right] \in Z . R$ is 2-torsionfree, so it follows that

$$
c x+u \in Z,
$$

where $u$ denotes the element $\left[B\left(x, x^{2}\right), x\right]$. In particular, $c x+u$ commutes with $x$, which implies that $u$ and $x$ commute.

Replacing $y$ by $x$ and $x$ by $x^{2}$ in (17), we obtain

$$
\left[B\left(x^{2}, x^{2}\right), x\right]+2\left[B\left(x^{2}, x\right), x\right] x+2 x\left[B\left(x^{2}, x\right), x\right] \in Z .
$$

Since $B$ is symmetric, this relation can be written as $v+2 u x+2 x u \in Z$ where $v=\left[B\left(x^{2}, x^{2}\right), x\right]$. However, $u$ and $x$ commute, so we have

$$
v+4 u x \in Z \text {. }
$$

In particular, $v+4 u x$ commutes with $x$; hence we see that $v$ and $x$ commute. Hence the element $2 x v=v x+x v=\left[B\left(x^{2}, x^{2}\right), x^{2}\right]$ lies in $Z$ by assumption. Therefore

The relation (18) yields

$$
0=[B(x, x), c x+u]=c[B(x, x), x]+[B(x, x), u] .
$$

Thus

$$
[B(x, x), u]=-c^{2} .
$$

According to (19) we have

$$
\begin{aligned}
0 & =[B(x, x), v+4 u x] \\
& =[B(x, x), v]+4[B(x, x), u] x+4 u[B(x, x), x] ;
\end{aligned}
$$


applying (21) we then get

$$
[B(x, x), v]=4 c^{2} x-4 c u .
$$

By (20),

$$
0=[B(x, x), v x]=[B(x, x), v] x+v[B(x, x), x]=[B(x, x), v] x+c v
$$

Using this relation and (22) we obtain

$$
4 c u x=4 c^{2} x^{2}+c v .
$$

Consider $w=[B(x, x), 4 c u x]$. We have

$$
w=4 c[B(x, x), u x]=4 c[B(x, x), u] x+4 c u[B(x, x), x],
$$

and so it follows from (21) that

$$
w=-4 c^{3} x+4 c^{2} u
$$

On the other hand, according to (23) we have

$$
\begin{aligned}
w & =\left[B(x, x), 4 c^{2} x^{2}+c v\right] \\
& =4 c^{2}([B(x, x), x] x+x[B(x, x), x])+c[B(x, x), v] \\
& =4 c^{2}(2 c x)+c[B(x, x), v],
\end{aligned}
$$

which together with (22) gives $w=12 c^{3} x-4 c^{2} u$. Comparing this expression with (24) one obtains $8 c^{2} u=16 c^{3} x$; thus $c^{2} u=2 c^{3} x$. Hence it follows from (21) that

$$
c^{4}=-\left[B(x, x), c^{2} u\right]=-\left[B(x, x), 2 c^{3} x\right]=-2 c^{3}[B(x, x), x]=-2 c^{4} .
$$

Thus $3 c^{4}=0$ and so $c^{4}=0$ since $R$ is 3-torsionfree. By assumption, this relation implies that $c=0$. This proves the theorem.

\section{ACKNOWLEDGMENT}

I would like to thank Professor J. Vukman for helpful conversations.

\section{REFERENCES}

1. H. E. Bell and W. S. Martindale, Centralizing mappings of semiprime rings, Canad. Math. Bull. 30 (1987), 92-101.

2. 500-508.

3. M. Brešar, Centralizing mappings on von Neumann algebras, Proc. Amer. Math. Soc. 111 (1991), 501-510.

4. __ Centralizing mappings and derivations in prime rings, preprint.

5. M. Brešar and J. Vukman, On some additive mappings in rings with involution, Aequationes Math. 38 (1989), 178-185.

6. I. N. Herstein, Rings with involution, Univ. of Chicago Press, Chicago, 1976.

7. C. Lanski, Differential identities, Lie ideals, and Posner's theorems, Pacific J. Math. 134 (1988), 275-297.

8. W. S. Martindale, Prime rings satisfying a generalized polynomial identity, J. Algebra 12 (1969), 576-584.

9. J. Mayne, Centralizing mappings of prime rings, Canad. Math. Bull. 27 (1984), 122-126. 
10. E. Posner, Derivations in prime rings, Proc. Amer. Math. Soc. 8 (1957), 1093-1100.

11. J. Vukman, Commuting and centralizing mappings in prime rings, Proc. Amer. Math. Soc. 109 (1990), 47-52.

University of Maribor, PF, Department of Mathematics, Koroška 160, 62000 Maribor, YugosLaVia 